EMERITA. Revista de Lingüística y Filología Clásica (EM)

LXXV 1, enero-junio de 2007

pp. $93-112$

ISSN 0013-6662

\title{
TEXTKRITISCHES ZU DEN EPIGRAMMEN MELEAGERS (I)
}

THOMAS GÄRTNER

Univ. Köln

In dem Aufsatz wird eine Vielzahl textkritischer Einzelprobleme aus den Epigrammen Meleagers besprochen in beständiger Auseinandersetzung mit der kommentierten Ausgabe von Gow und Page.

Schlüsselwörter: Meleager; Epigrammatik; Textkritik; Anthologia Palatina
In the present paper a number of questions of textual criticism concerning the epigrams of Meleager is raised. The commented edition of Gow and Page is constantly used as a starting point of the discussion.

Keywords: Meleager; epigrams; textual criticism; Anthologia Palatina

Meleager AP IV 1, 1 - 4 = HE 3926 - 3929

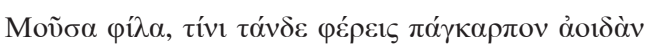

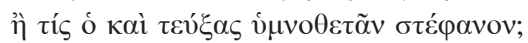

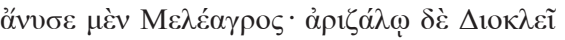

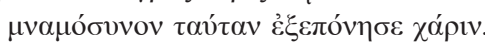

Das Partizip $\tau \varepsilon v \xi^{\prime} \alpha \varsigma$ erweckt den falschen Eindruck, Meleager habe einen eigenen Kranz 'geschaffen'. Tatsächlich 'wand' er einen solchen aus den poetischen Erzeugnissen Früherer:

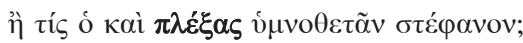

Vgl. das Schlußepigramm, AP XII 257 = HE $4722-4729$

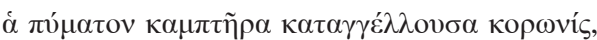

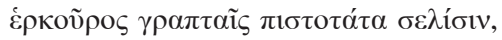

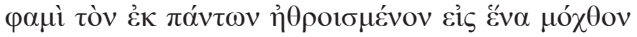

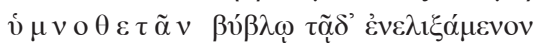

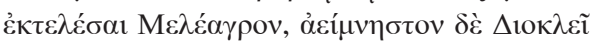

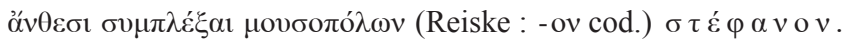

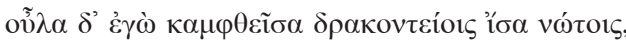

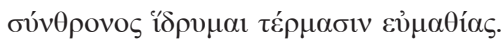

$$
\text { AP V } 176=\text { HE } 4022-4027
$$

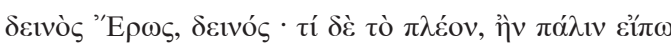

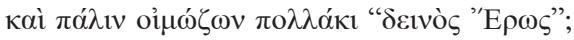




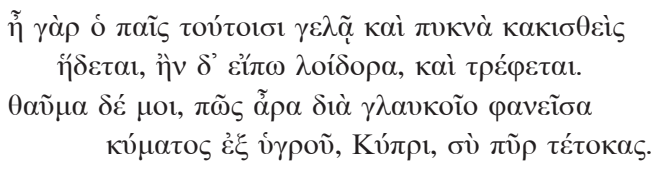

Obwohl $\tilde{\alpha} \rho \alpha$ statt $\alpha$ $\rho \alpha$ nach Fragepronomen (Denniston 45 f.) auch sonst und insbesondere in hellenistischer Dichtung (Gow zu Theocr. 7, 105) tadel-

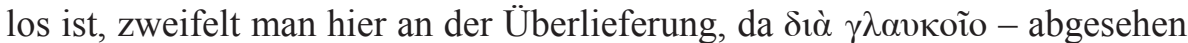
von кú $\mu \alpha \tau o \varsigma$ im folgenden Vers - keinerlei Bezugswort hat. Man erwartet einen Rekurs auf die Schaumgeburt der Aphrodite, der sich etwa folgendermaßen einführen ließe:

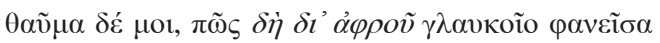

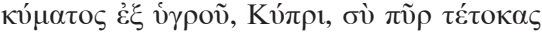

Zum Gebrauch von $\delta \eta ́$ in indirekten Fragesätzen vgl. Denniston 211 f.

$$
\begin{aligned}
& \text { AP V } 179=\text { HE } 4028-4037
\end{aligned}
$$

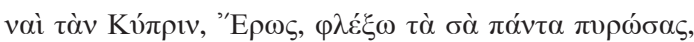

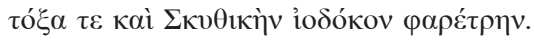

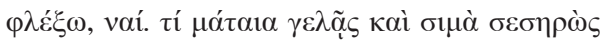

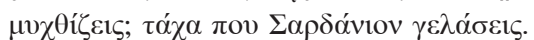

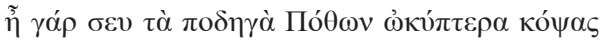

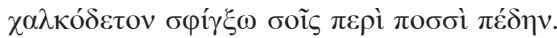

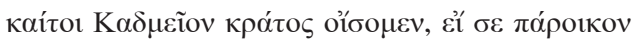

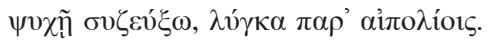

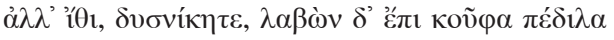

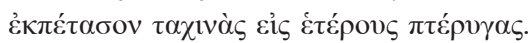

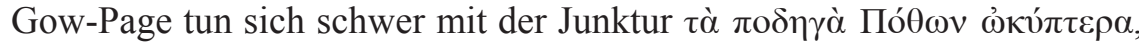
die sie im Anschluß an Graefe («alae Amoris adducunt Cupidines, i.e. advolans Amor Cupidines secum adducit») auf andere Liebesgottheiten beziehen, von denen der hier singularisch auftretende Eros begleitet werden sollte. Um solche bizarren Vorstellungen kommt man herum, wenn man $\pi$ ó $\theta \omega v$ mit kleinem Anfangsbuchtsaben schreibt und als Abstractum versteht: Die Flügel des Eros "bereiten den Liebessehnsüchten den Weg" bzw. "leiten" diese

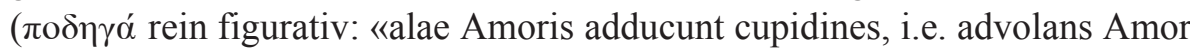
cupidines secum adducit»), und gerade deshalb will der (von unglücklicher

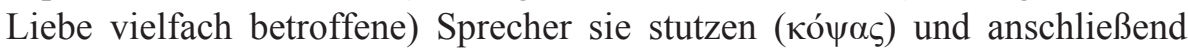
Eros fesseln. Die richtige Erklärung erwog bereits Jacobs: «alas cupiditaes et desiderium adferentes». Im übrigen könnte man, wenn den Begriff $\pi \circ \delta \eta \gamma \alpha ́$

$$
\text { EMERITA (EM) LXXV 1, enero-junio } 2007 \quad \text { pp. 93-112 ISSN 0013-6662 }
$$


(wörtlich 'fußleitend') in bezug auf 'Flügel' schwierig findet, eher als Stadtmüllers $\tau \alpha \dot{\delta} \delta$ ' ỏ $\pi \eta \delta \alpha$ ein $\tau \alpha \dot{\delta} \delta^{\prime}$ ó $\delta \eta \gamma \alpha$ für möglich halten, worin 'Weg' und

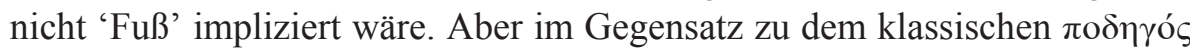

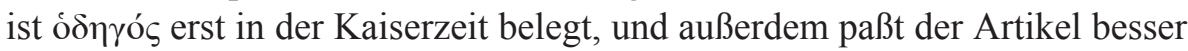
als das Demonstrativpronomen zum Possessivum $\sigma \varepsilon v$, so daß man bei den überlieferten "fußleitenden Flügeln" bleiben sollte. Wenn diese die Form von Flügelschuhen haben (wie das Schlußdistichon zu implizieren scheint), ist das auf den ersten Blick schwierige Bild ("die Flügel des Eros leiten den Fuß der Begierden") einigermaßen verständlich.

Im nächsten Distichon fällt dem Sprecher ein, daß ein nach seinem Plan flugunfähig gemachter und gefesselter Eros ja auf immer bei ihm verharren müßte, und läßt ihn doch wegfliegen. Dieses Motiv greift Ovid im Prooemium zum zweiten Buch seiner Ars amatoria auf, wendet es aber entscheidend anders: Die "Fesselung" des Liebesgotts ist dort keine strafende Gewalthandlung, sondern die systematische Fixierung eines einmal initiierten Liebesverhältnisses; sie bildet eine - anders als im vierten Distichon des hier besprochenen Gedichts - zwar erstrebenswerte, aber äußerst schwierige Aufgabe.

Das Motiv, daß dem Eros die Flügel gestutzt werden, begegnet zuerst bei Aristophon fr. 11 K.-A.:

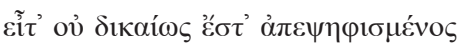

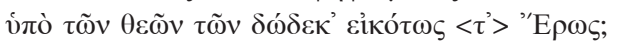

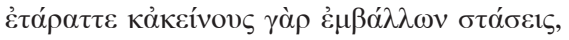

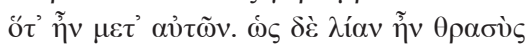

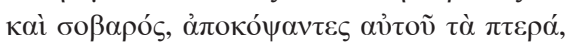

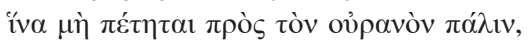

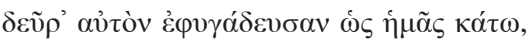

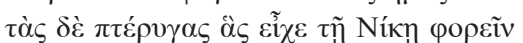

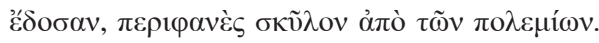

Man erkennt deutlich die Entwicklung des Motivs: Zunächst handelt es sich um ein sophistisches Aition für die Liebe in der Menschenwelt, innerhalb dessen die Stutzung der Flügel natürlicherweise nur von den Göttern geleistet werden kann. In der hellenistischen Epigrammatik wird es von den Göttern auf ein verzweifeltes dichterisches Ich übertragen, in der Gestalt einer gewaltsamen Strafandrohung. Bei Ovid schließlich ist die Fesselung Amors ein vom Liebeslehrer gelehrter schwieriger technischer Akt, der die göttliche Macht der Liebe menschlicher Rationalität zugänglich machen soll. 
Das Epigramm ist wohl auf dem Wege einer Motivumkehrung direkt aus dem aitiologischen Mythos herausentwickelt: Während die Götter Eros

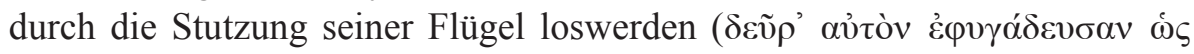

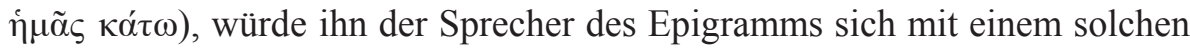

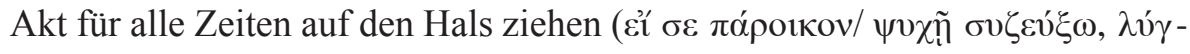
$\kappa \alpha \pi \alpha \rho^{\prime} \alpha i \pi \mathrm{i} \lambda i_{0}(\varsigma)$.

Eine weitere Fortentwicklung findet das Motiv bei Paul. Sil. AP V 268: Eros stutzt sich die Flügel selbst, um bei dem Sprecher bleiben zu können und ihn zu quälen:

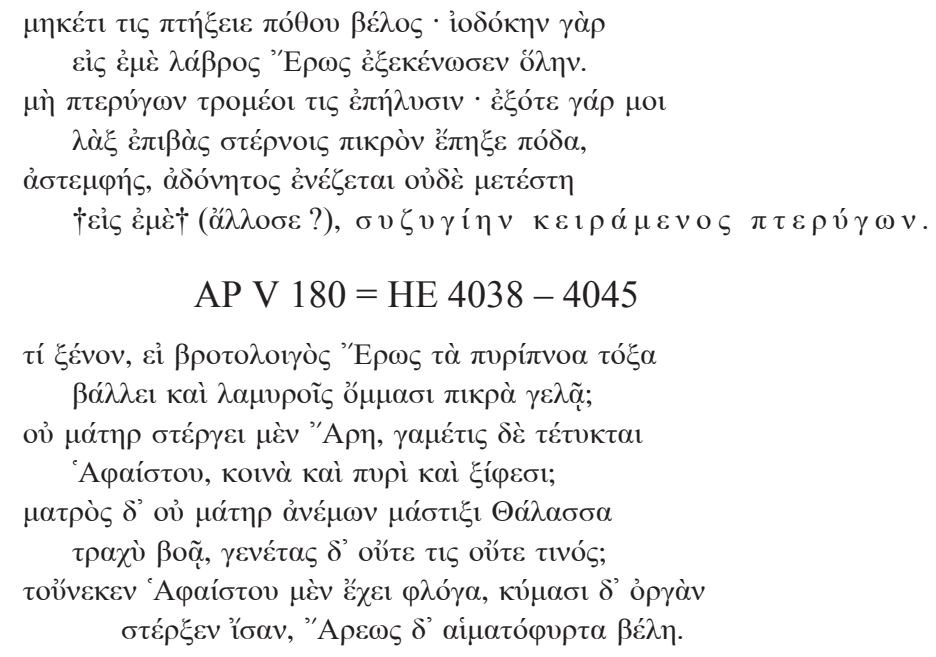

Gow-Page erklären $\sigma \tau \varepsilon \dot{\rho} \rho \xi \varepsilon v$ als «fovet». $\sigma \tau \varepsilon ́ \rho \gamma \varepsilon \imath v$ muß jedoch immer mit abstrakten Objekten verbunden sein und hat stets die Nuance «sich innerlich mit etwas zufriedengeben», «acquiesce» (LSJ s.v. III 2). In diesem Sinne

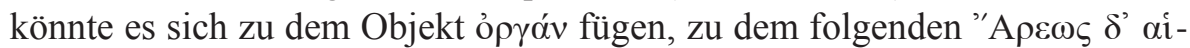
$\mu \alpha \tau$ ó gänzen. Mit einem neuen Praedikat im zweiten Glied hätte Meleager sich stilistisch kaum einen Gefallen getan.

Zudem erwartet man aber auch eine inhaltliche Füllung von кú $\mu \alpha \sigma \iota \delta$

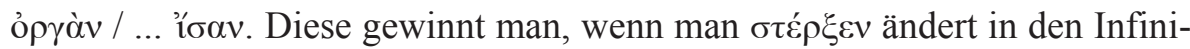
tiv $\sigma \pi \varepsilon \dot{\varepsilon} \rho \xi_{\alpha 1}$ (Waltz versuchte $\sigma \pi \varepsilon \dot{\varepsilon} \rho \xi \varepsilon v$, was Gow-Page in dieser Form zurecht

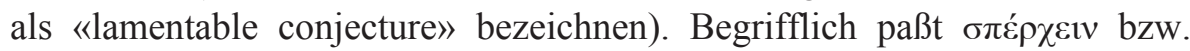
$\sigma \pi \varepsilon ́ p \chi \varepsilon \sigma \theta \alpha 1$ sehr gut einerseits zu Naturgewalten (LSJ s.v. II), andererseits 
zu aufbegehrenden Gefühlsinstanzen (LSJ s.v. I 2). Insbesondere das Aktiv

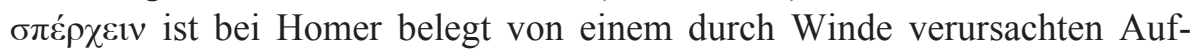

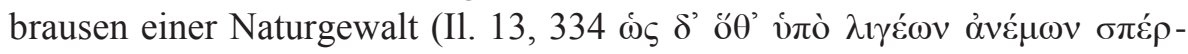
$\chi \omega \sigma \iota v$ á $\varepsilon \lambda \lambda \alpha \mathrm{l})$, so daß das Wort gut auf das von den peitschenden Winden aufgewühlte Meer (5 f.) paßt. Den schwachen Aorist von $\sigma \pi \varepsilon \dot{\varepsilon} \rho \varepsilon ı v$ (im Me-

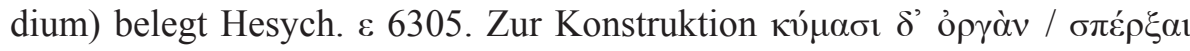

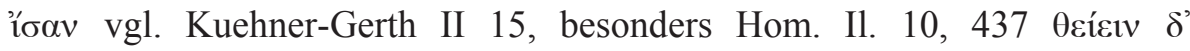

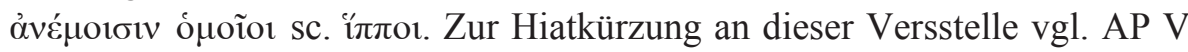

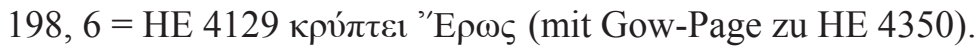

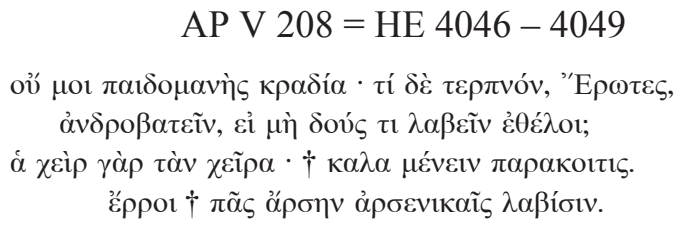

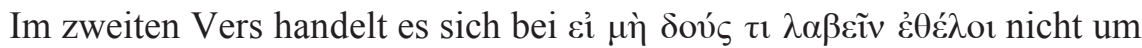
eine Bedingung des $\alpha \dot{\alpha} \delta \rho \rho \beta \alpha \tau \varepsilon \tilde{i} v$, sondern um einen notwendig stets damit

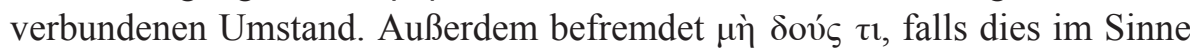

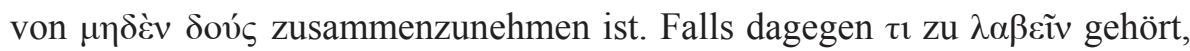
stößt man sich am Fehlen eines Objekts zu $\mu \grave{j} \delta$ ov́s. In Anbetracht der termi-

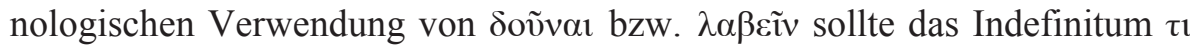
ohnehin besser wegbleiben. Insofern wäre die Fassung

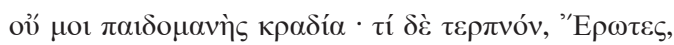

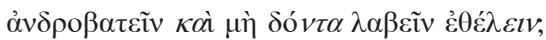

sprachlich wesentlich glatter. Überliefert ist nach $\alpha \dot{\alpha} \delta \rho \rho \beta \alpha \tau \varepsilon i v v$ genaugenommen $\tilde{\eta}$, nicht $\varepsilon i$. Sachlich hat die Kritik am homosexuellen Liebesakt, welcher nicht auf gleichmäßigem "Geben und Nehmen", also beiderseitiger Befriedigung, beruht, eine enge Parallele bei Ov. ars II 682 - 684 (vgl. Jankas Kommentar z.St.):

Quod iuuat, ex aequo femina uirque ferant.

Odi concubitus, qui non utrumque resoluunt: Hoc est, cur pueri tangar amore minus.

Was Vers 3 anbelangt, so referieren Gow-Page (von den vielen bei Stadt-

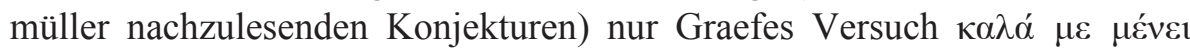

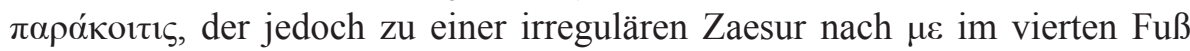


führe. An den Versuchen im Schlußvers monieren Gow-Page, daß immer der bloße Dativ in dem Sinne «together with your masculine embraces» verstanden werden müßte. Beide Schwierigkeiten würden umgangen mit folgender Fassung:

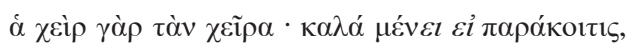

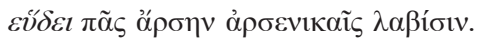

"Wenn eine schöne Bettgenossin wartet, schläft jeder Mann (d.h. ist unempfänglich) für männliche Umarmungen". Zur Hiatkürzung nach zweisilbigem Wort an der Stelle der bukolischen Dihaerese bei Meleager vgl. Gow-Page

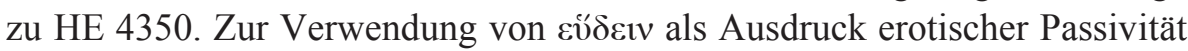
vgl. AP V 152, 3 f. = HE 4176 f. (wo ein etwas anderer Zusammenhang mit

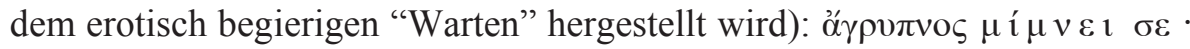

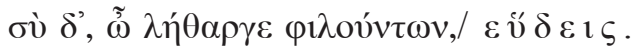

$$
\begin{aligned}
& \text { AP V } 212=\text { HE } 4050-4055
\end{aligned}
$$

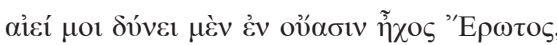

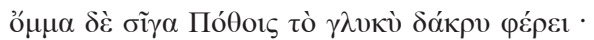

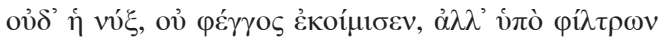

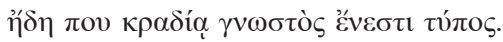

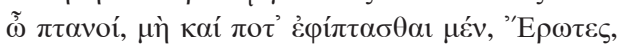

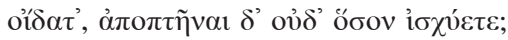

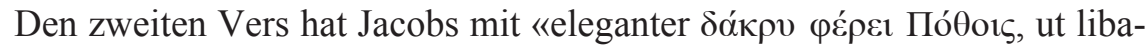
tionem Amoribus oblatam» vorzüglich erklärt (trotz Gow-Pages Widerspruch: «far-fetched, rather, and too elaborate»). Man sollte aber den Begriff "ut libationem" anstelle des nicht recht passenden $\sigma \tilde{i} \gamma \alpha$ in den Text bringen:

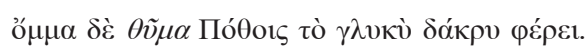

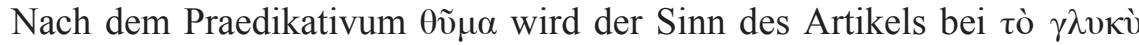

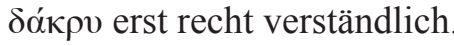

$$
\text { AP XII } 117=\text { HE } 4092-4097
$$

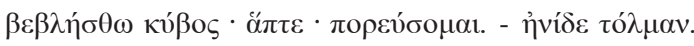

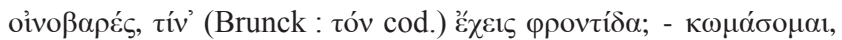

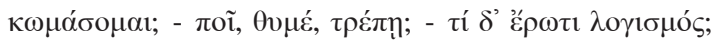

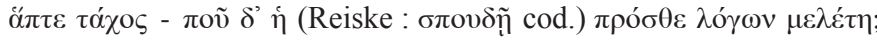

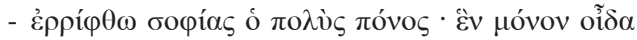

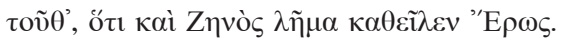


Gow-Page erwägen, die von Brunck gemäß einem Apographon herge-

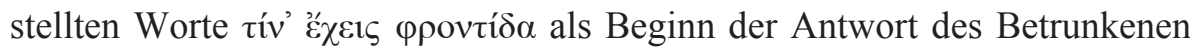
zu fassen («what are you worried about?»). So würden freilich die nicht

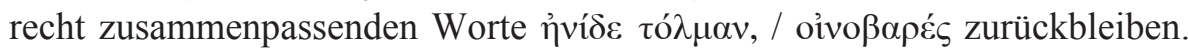
Aber auch die schließlich von Gow-Page favorisierte Auffassung als Anrede an den Betrunkenen in der Bedeutung "what thought have you in mind? i.e.

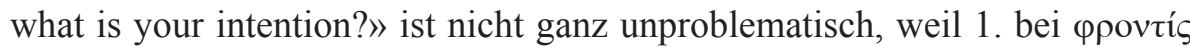

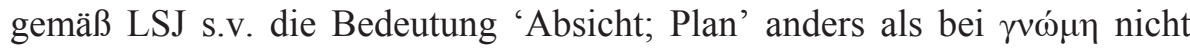

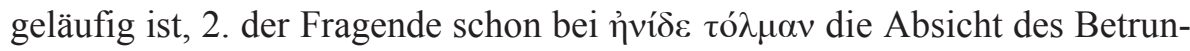
kenen verstanden zu haben scheint, also nach etwas ihm Bekanntem fragen würde, und 3. die Ingeminatio $\kappa \omega \mu \alpha ́ \sigma o \mu \alpha 1, / \kappa \omega \mu \alpha ́ \sigma o \mu \alpha \iota$ eher wie ein trotziges Beharren als wie eine Antwort auf eine Informationsfrage wirkt. Besser

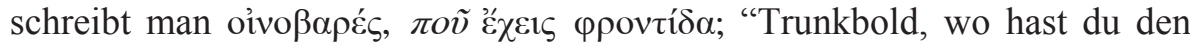

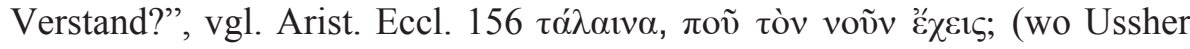
ad loc. kaum zurecht die Bedeutung «where have your wits gone?» ablehnt zugunsten von «what are you thinking of? ... her mind has been wandering») Dann gehen die drei an den Betrunkenen gerichteten Fragen alle in die gleiche Richtung, nämlich seine Verstandlosigkeit zu kritisieren.

$$
\begin{aligned}
& \text { AP XII } 132 \text { b = HE } 4110-4117
\end{aligned}
$$

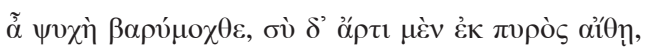

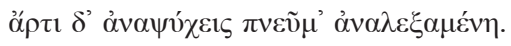

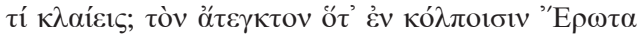

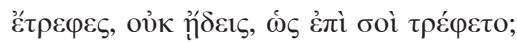

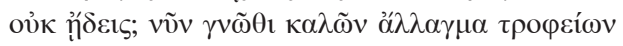

$$
\begin{aligned}
& \pi \tilde{\nu} \rho \not \alpha \mu \alpha \kappa \alpha i ̀ ~ \psi v \chi \rho \alpha ̀ v ~ \delta \varepsilon \xi \alpha \mu \varepsilon ́ v \eta ~ \chi 1 o ́ v \alpha .
\end{aligned}
$$

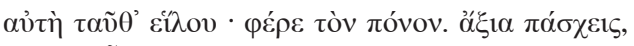

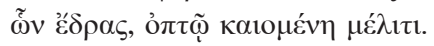

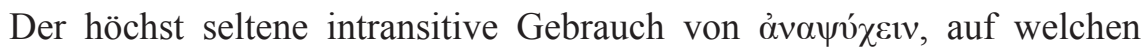
sich Gow-Page berufen, ist äußerst fragwürdig in einem Zusammenhang, wo man nach aîn ein zweites Passiv erwartet, zumal es nach dem sechsten Vers

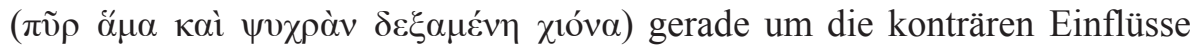
geht, denen die Seele durch Eros ausgesetzt wird. Also wird man keine Be-

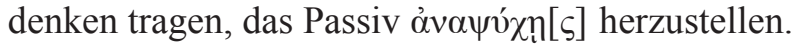

Die von Gow-Page als Neuheit bezeichnete Metapher $\pi v \varepsilon \tilde{v} \mu$ ' $\alpha v \alpha \lambda \varepsilon \xi \alpha$ $\mu \varepsilon \dot{v} \eta$ kann man jetzt mit Hilfe des Mailänder Epigrammpapyros auf ihr 
wahrscheinliches Vorbild zurückführen, epigr. 95, 1 - 4 A.-B. = col. IV 30 33 (von der Abbildung eines genesenden Kranken):

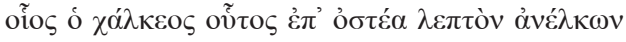

$$
\begin{aligned}
& \pi v \varepsilon \tilde{u} \mu \alpha \mu o_{\gamma}[\zeta] \zeta \omega \eta ̀ v ~ o ̈ \mu \mu \alpha \tau \iota ~ \sigma v \lambda \lambda \varepsilon ́ \gamma \varepsilon \tau \alpha 1,
\end{aligned}
$$

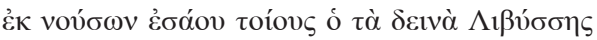

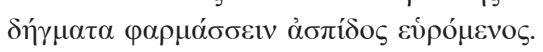

Mit $\pi v \varepsilon \tilde{v} \mu^{\prime} \dot{\alpha} v \alpha \lambda \varepsilon \xi \alpha \mu \varepsilon \varepsilon^{2} \eta$ wird der komplexe Ausdruck $\dot{\varepsilon} \pi^{\prime}$ ỏ $\sigma \tau \varepsilon \dot{\varepsilon} \alpha \lambda \varepsilon \pi \tau o ̀ v$ $\dot{\alpha} v \varepsilon \dot{\varepsilon} \kappa \omega v / \pi v \varepsilon \tilde{v} \mu \alpha \mu$ ó $\gamma[\zeta] \zeta \omega \eta ̀ v ~ o ́ \mu \mu \alpha \tau \imath ~ \sigma v \lambda \lambda \dot{\varepsilon} \gamma \varepsilon \tau \alpha \iota$ zu einer kompakten Junktur komprimiert.

$$
\text { AP V } 198=\text { HE } 4124-4129
$$

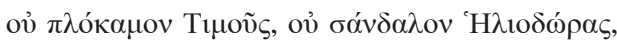

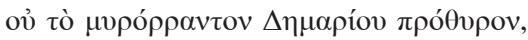

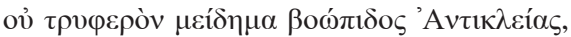

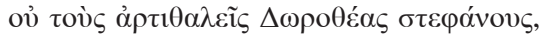

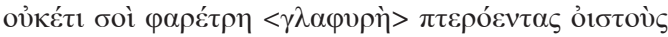

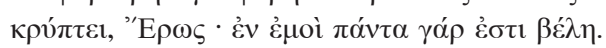

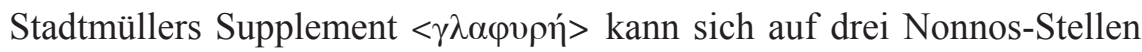
(Dion. 13, 307; 47, 583; 48, 625) stützen. Erstaunlicherweise wird auch von keinem der bei Stadtmüller und Gow-Page genannten Versuche ein im Sinne der Gesamttendenz der Partie effektives Epitheton hergestellt. Daß sich die

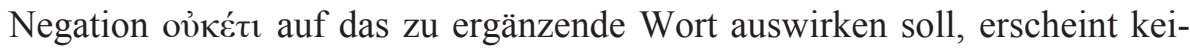
neswegs sicher. Möglich wäre etwa

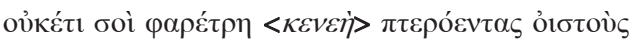

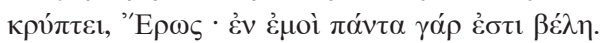

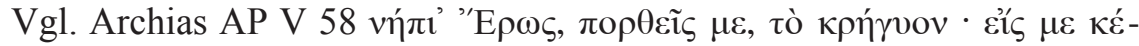

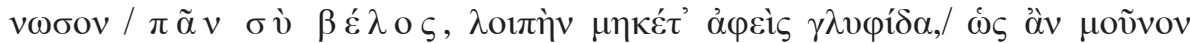

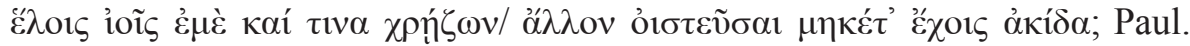

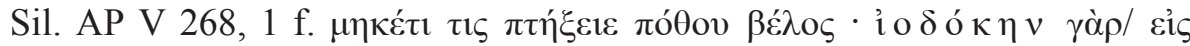

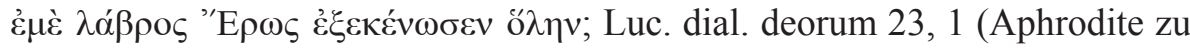

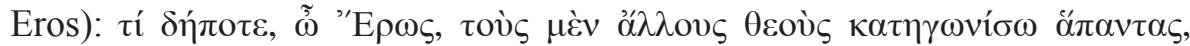

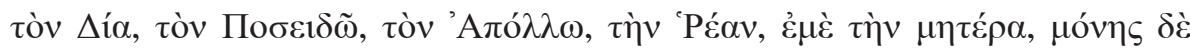

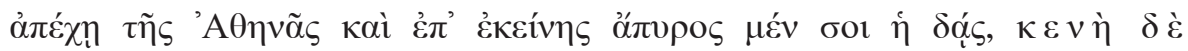

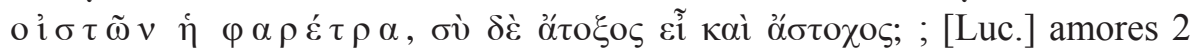

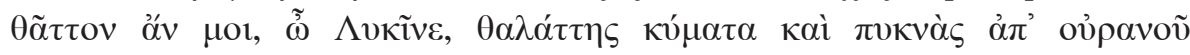

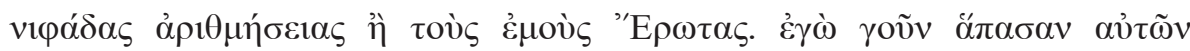

$$
\text { EMERITA (EM) LXXV 1, enero-junio } 2007 \quad \text { pp. 93-112 ISSN 0013-6662 }
$$




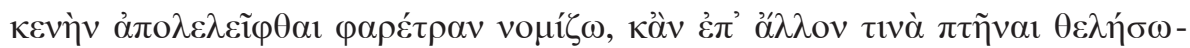

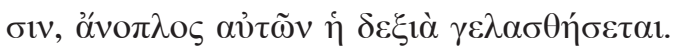

$$
\text { AP V } 160=\text { HE } 4132-4135
$$

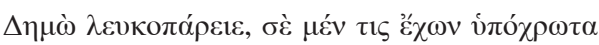

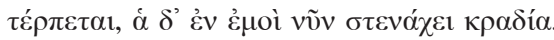

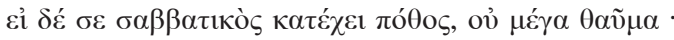

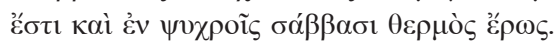

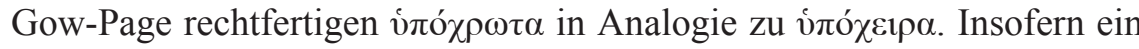
solches Adjektiv aber nirgends belegt und auch ein "̌́ $\chi \omega v$ vं $\pi$ ò $\chi \rho \tilde{\omega} \tau \alpha$ bzw.

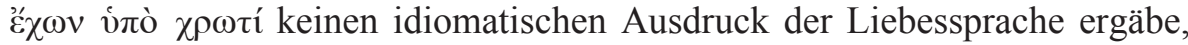
wird man "̌ $\chi \omega v$ í̃ò $\chi \lambda \alpha$ íva (Graefe) schreiben müssen, vgl. Soph. Trach.

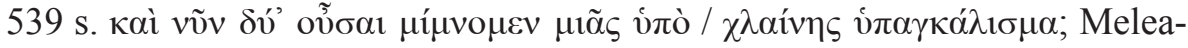

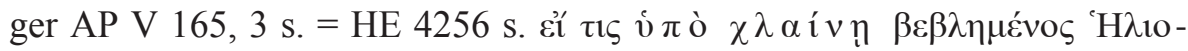

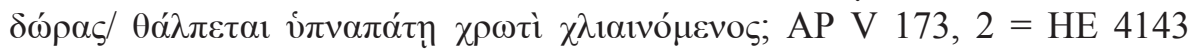

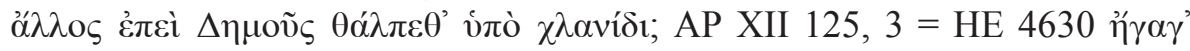

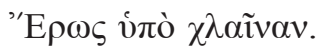

$$
\text { AP V } 172 \text { = HE } 4136-4141
$$

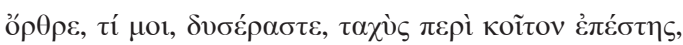

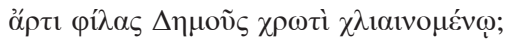

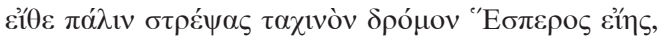

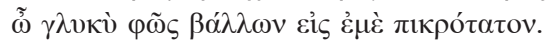

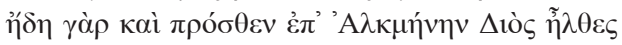

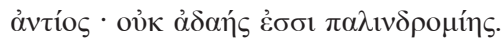

Gow-Page halten die Überlieferung, aber der Sinn «you went backwards to Zeus's Alcmena» wird niemanden befriedigen. Eine Abänderung in '̇ं $\pi^{\prime}$

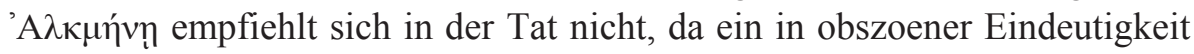

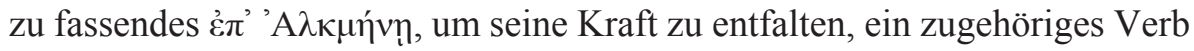

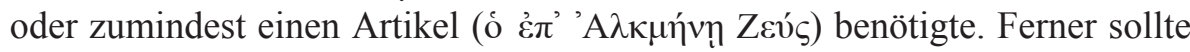
man auch nicht den Genitiv $\Delta$ iós mit ảvtíos zusammennehmen, da es nicht darumgeht, Zeus "entgegenzutreten", und außerdem der Verbalausdruck $\tilde{\eta} \lambda$ -

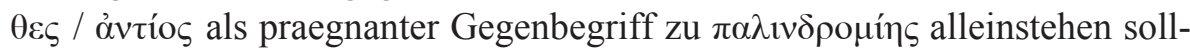
te. Andererseits ist aber auch die Junktur 'A $\lambda \kappa \mu \eta \dot{v \eta \eta} v$ iò $\varsigma$ in dem Sinne "Alkmene, die Geliebte des Zeus" nicht idiomatisch (vgl. Gow-Page z.St.).

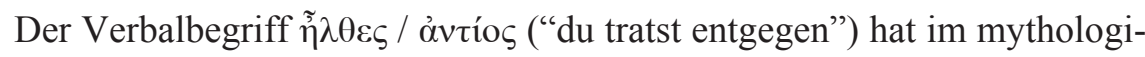

$$
\text { EMERITA (EM) LXXV 1, enero-junio } 2007 \quad \text { pp. 93-112 ISSN 0013-6662 }
$$


schen Zusammenhang der Alkmene-Geschichte offenkundig nur in negierter Form Platz. Erwägenswert wäre die Herstellung einer rhetorischen Frage der Art

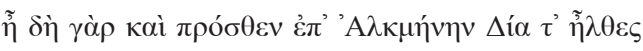

$$
\begin{aligned}
& \text { ảvtíos; ... }
\end{aligned}
$$

"Bist du fürwahr denn etwa auch vormals Alkmene und Zeus entgegengetreten?". Dann läge eine (durchaus plausible) Kombination aus affirmativem $\tilde{\eta}$ $\delta \eta ́$ (Denniston 285) und fragendem $\eta^{\tilde{n}}$... $\gamma \grave{\alpha} \rho$ (Denniston 284 f.) vor. Eindeutigen negativen Sinne ergäbe dagegen die Textfassung

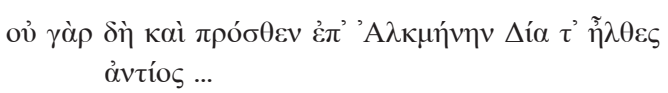

"Denn fürwahr bist du nicht auch vormals Alkmene und Zeus entgegengetre-

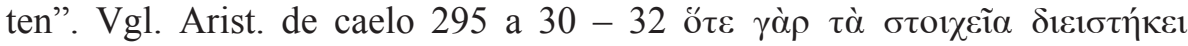

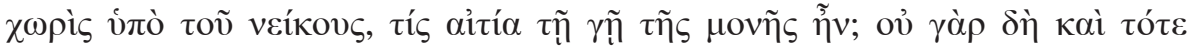

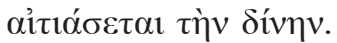

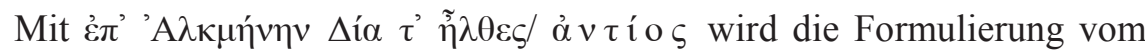

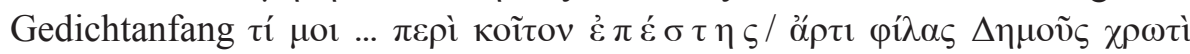

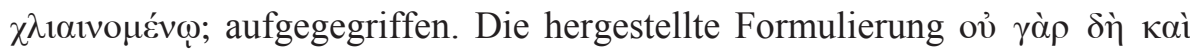
$\pi \rho \sigma^{\sigma} \theta \varepsilon v$ reflektiert darauf, daß es im jetzigen Fall anders ist, mit oủ $\delta \dot{\varepsilon}$ yò $\rho$ $\delta \grave{\eta} \pi \rho o ́ \sigma \theta \varepsilon v$ wäre dagegen eine Analogie des früheren Falls zu dem jetzigen impliziert.

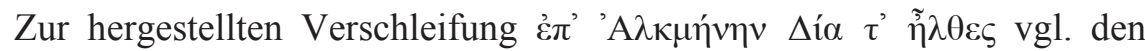
Versschluß AP XII 125, $5=$ HE 4632 ö $\mu \mu \alpha \sigma ı ~ \delta ' v ̈ \pi v o v$. Die konjektural hergestellte negative Valenz des mythischen Exempels bestätigt sich durch das eng verwandte Epigramm Makedonios AP V 223:

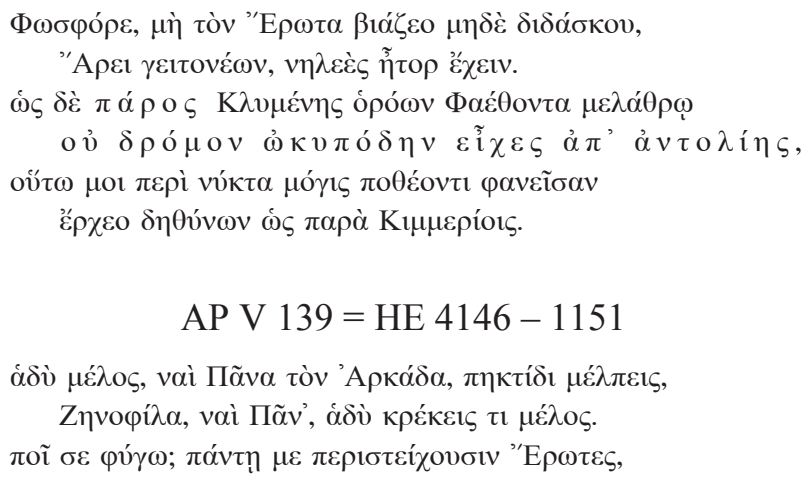

EMERITA (EM) LXXV 1, enero-junio $2007 \quad$ pp. 93-112 ISSN 0013-6662 


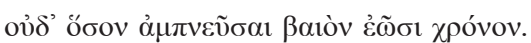

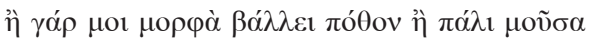

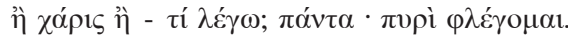

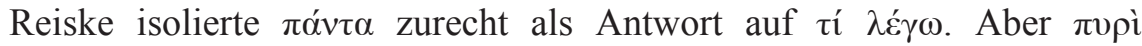
$\varphi \lambda \varepsilon ́ \gamma o \mu \alpha$ bildet keine befriedigende Schlußpointe. Eine solche gewinnt man erst mit $\pi \varepsilon \rho \iota \varphi \lambda \varepsilon ́ \gamma o \mu \alpha 1$, vgl. Polyb. XII 25, 2 (vom Stier des Phalaris) ஸ̋ $\sigma \tau^{\prime}$

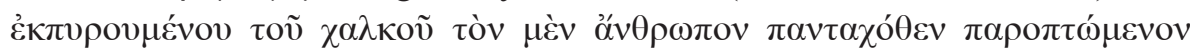

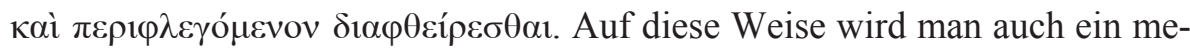
trisches Problem los, nämlich die Längung eines kurzen Vokals am Wortende vor anlautender Muta-Liquida-Kombination, die bei Meleager nur unter bestimmten Bedingungen zulässig ist (vgl. Gow-Page z.St.).

$$
\begin{aligned}
& \text { AP V } 140=\text { HE } 4152-4155
\end{aligned}
$$

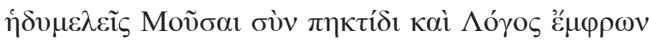

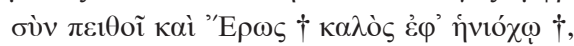

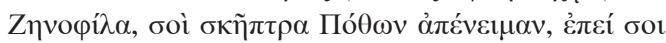

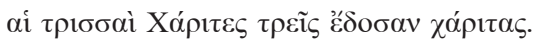

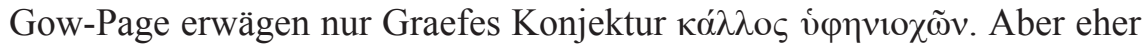
als das müßige Praefix von v́

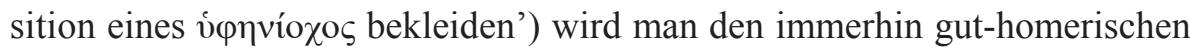

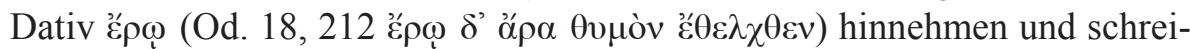
ben:

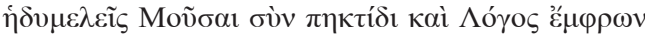

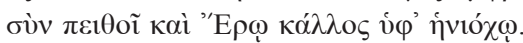

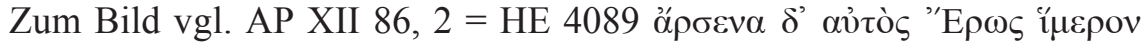

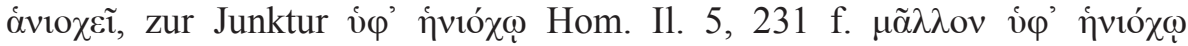

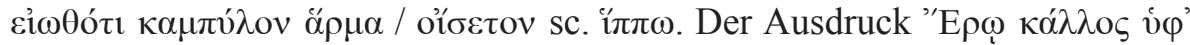

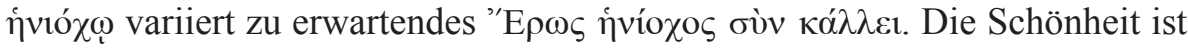
genauso das Instrument des Eros wie die $\pi \eta \kappa \tau i ́ \varsigma$ dasjenige der Musen und die Überzeugung dasjenige des Logos. Dann ist in allen drei Gliedern (anders

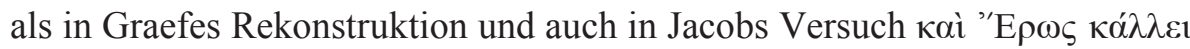
$\sigma \grave{v} \gamma \lambda \lambda v \kappa \varepsilon \rho \tilde{\omega})$ die leitende Instanz mit einem Attribut bekleidet.

$$
\text { AP V } 152=\text { HE } 4174-4181
$$

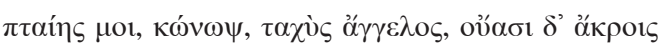

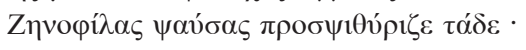




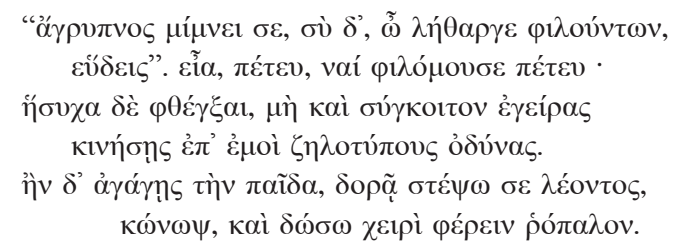

Das Adjektiv $\varphi \imath \lambda o ́ \mu o v \sigma o \varsigma$ ist bei Meleager singulär, und man begreift auch sachlich nicht, warum die Mücke als 'Musenfreund' bezeichnet werden sollte, es sei denn, Meleager verstünde sich an dieser Stelle nicht als gequälten Liebhaber, sondern als Dichter (Giangrande, Mnem. 25, 1972, s. 297 f.

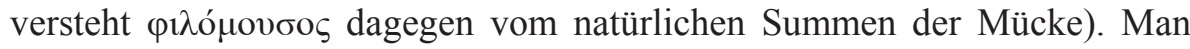
wird ein bei Meleager häufiger vorkommendes Attribut einsetzen und

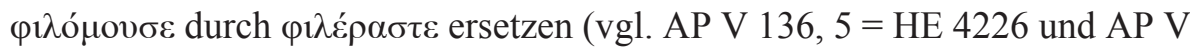
147, 4 = HE 4239 von Rosen, AP V 144, 3 = HE 4158 von Zenophila). Mit diesem Vokativ apostrophiert Meleager insbesondere in AP V 163, 5 f. $=\mathrm{HE}$ $4252 \mathrm{f}$. eine ebenfalls als Bote im Dienst des Liebenden verstandene Biene:

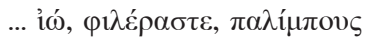

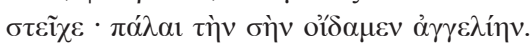

Mit dem auf die Mücke bezüglichen $\varphi \imath \lambda \varepsilon ́ p \alpha \sigma \tau \varepsilon$ erhält der auf die spröde

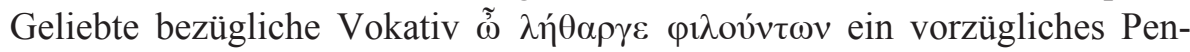
dant.

$$
\begin{aligned}
& \text { AP V } 177=\text { HE } 4190-4199
\end{aligned}
$$

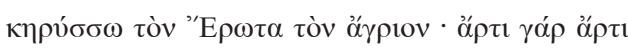

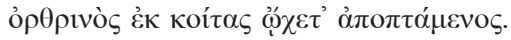

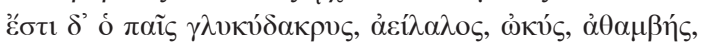

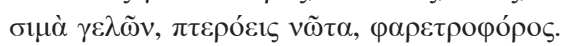

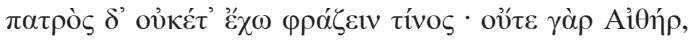

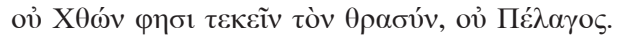

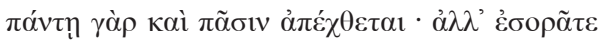

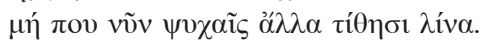

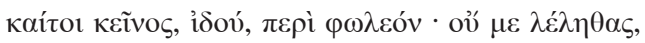

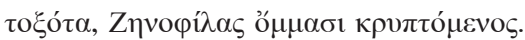

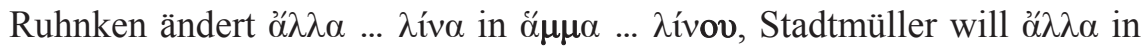
oṽ $\lambda \alpha$ ändern. Aber vor dem finalen Outing von Eros' tatsächlichem Aufent-

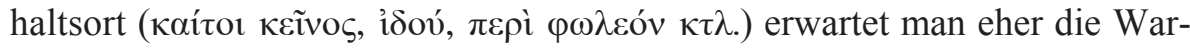
nung, daß Eros, nachdem er sich bei den Göttern "überall und jedem" verhaßt gemacht hat, irgendwo anders (in der Menschenwelt) ahnungslosen

$$
\text { EMERITA (EM) LXXV 1, enero-junio } 2007 \quad \text { pp. 93-112 ISSN 0013-6662 }
$$


Seelen "Netze" spannt:

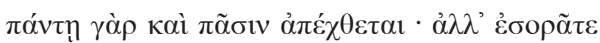

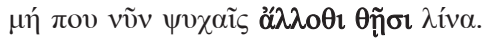

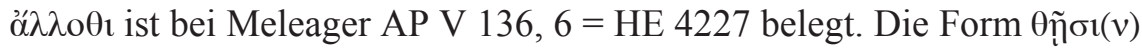

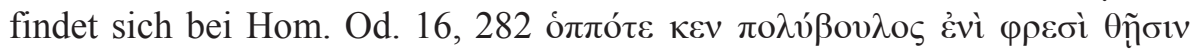
'A $\theta$ ńvๆ. Daß in negativen Finalsätzen nach Verben des Zusehens, «wo das Deutsche geneigt ist, $\mu$ ́ durch ob nicht zu übersetzen und damit die Andeutung der Besorgnis aufzugeben», auch der Konjunktiv geläufig ist, zeigen Kuehner-Gerth II 392.

$$
\text { AP V } 195=\text { HE } 4210-4215
$$

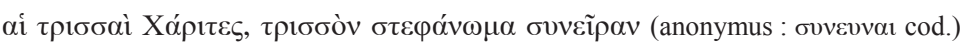

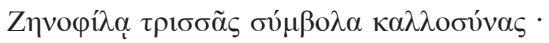

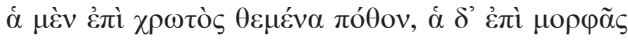

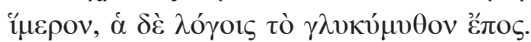

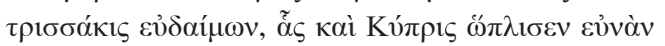

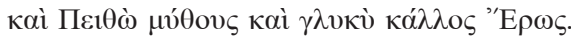

Mediales غ̇ंı $\dot{\theta} \theta \varepsilon \sigma \theta \alpha \iota$ kann kaum "einem anderen etwas beilegen" heißen. Das Gedicht entspricht strukturell dem Einleitungsgedicht zu Meleagers Kranz (AP IV 1 = HE 3926-3983, ähnlich auch der "Knabenkranz", AP XII 256 = HE 4408-4419): Am Anfang wird jeweils das Flechten eines Kranzes beschrieben, dann werden die Ingredienzen aufgezählt. In letzterem Zusammenhang ist mediales $\tau i ́ \theta \varepsilon \sigma \theta \alpha$ ("von sich aus beitragen") gebräuchlich (AP

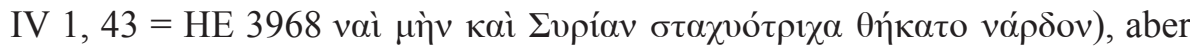
daneben muß als Praeposition nicht غ̇ंí, sondern das bei der Aufzählung von Ingredienzien obligatorische $\dot{\varepsilon} v$ stehen, das hier jedoch nicht anaphorisch ( $\dot{\varepsilon} v$ $\left.\mu \varepsilon^{\prime} v-\varepsilon \dot{\varepsilon} v \delta \dot{\varepsilon}\right)$ verwendet werden kann, weil es - anders als im Einleitungsgedicht - vor allem auf die verschiedene Identität der Beiträger ankommt:

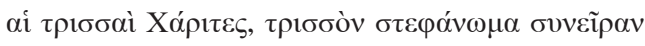

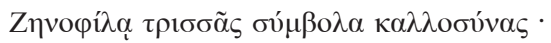

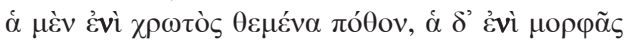

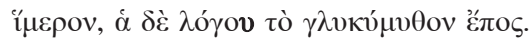

AP V $24=$ HE $4218-4221$

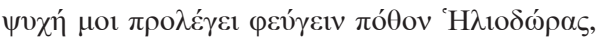

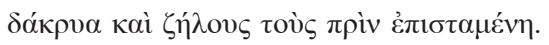

EMERITA (EM) LXXV 1, enero-junio $2007 \quad$ pp. 93-112 ISSN 0013-6662 


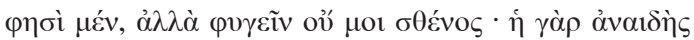

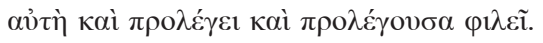

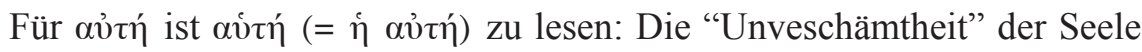
besteht darin, daß sie zugleich ("als dieselbe") kluge Mahnungen ausspricht und trotz dieser Mahnungen Heliodora liebt.

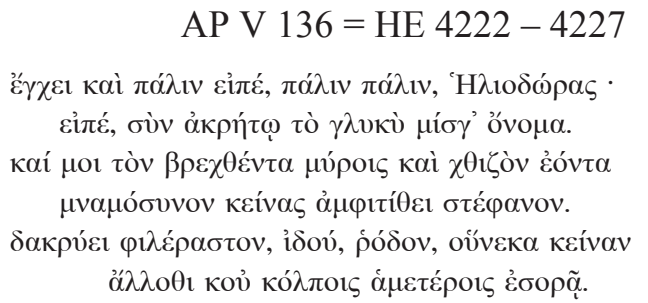

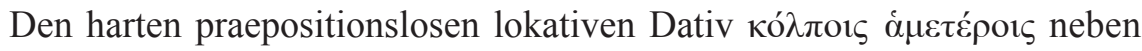
$\alpha_{\alpha} \lambda \lambda \mathrm{o} \theta \mathrm{r}$ kann man abstützen, indem man $\dot{\varepsilon} \sigma o \rho \tilde{a}$ mit geringfügigem Eingriff in

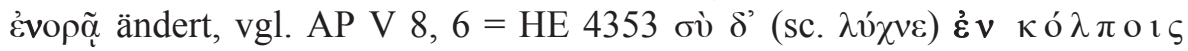
$\alpha \hat{v} \tau$ òv ó $\rho \tilde{\alpha} \varsigma \delta \dot{\varepsilon} \tau \dot{\varepsilon} \rho \omega v$. Das Praefix von $\dot{\varepsilon} v o \rho \tilde{\alpha} v$ bezieht sich idiomati-

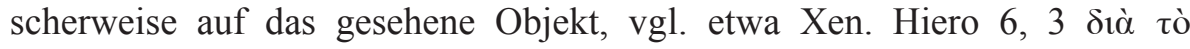

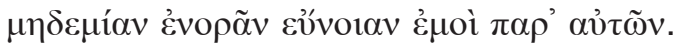

$$
\text { AP V } 137=\text { HE } 4228-4231
$$

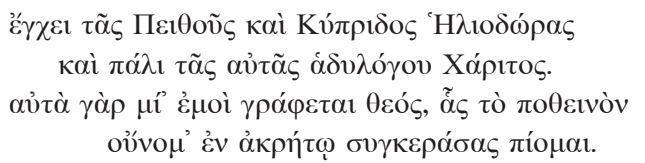

Gow-Page: "'she herself is reckoned one goddess' is an elliptic way of saying that the attributes of all three divinities are combined in her one person». Wie man dies ausdrücken kann, ohne den Begriff der Dreiheit auszusprechen, bleibt rätselhaft, zumal wenn der $\gamma \alpha \dot{\rho} \rho-$ Satz offenbar begründen muß, warum der Sprecher dreimal auf die eine Heliodora trinkt. Hinzu

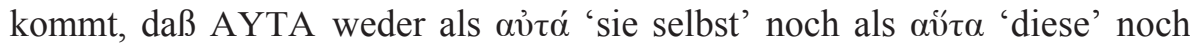
als $\alpha \dot{\tau} \alpha$ ' 'dieselbe' Sinn ergibt. Offenkundig liegt ein Influenzfehler aus $\tau \tilde{\alpha} \varsigma$

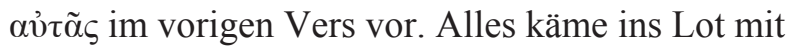

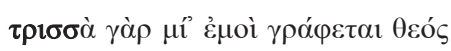

"Obwohl eine Person, rechne ich sie als dreifache Gottheit". Zum Spiel 


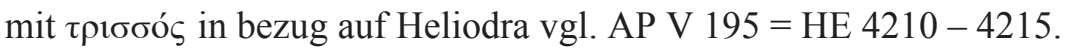

AP V 147 = HE $4236-4241$

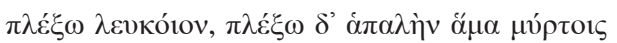

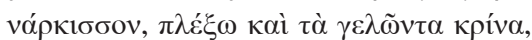

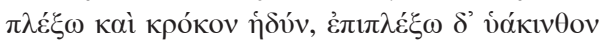

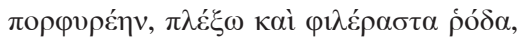

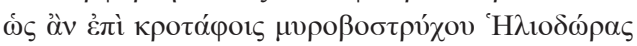

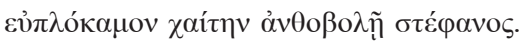

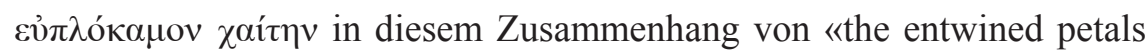
of the garland» zu verstehen (Gow-Page) geht kaum an; die Junktur muß

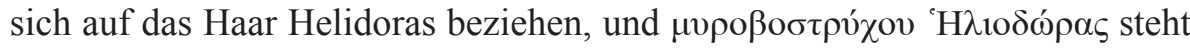

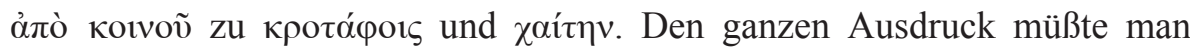
dann - gemäß kaiserzeitlichem Gebrauch von $\dot{\alpha} v \theta$ o $\beta$ o $\lambda \varepsilon \tilde{v}$ - so verstehen, daß der Kranz (mit seinen Blumen) Heliodoras Haar durch "Blumenwurf" verherrlichen soll wie die Menge (mit Blumen und Kränzen) einen siegrei-

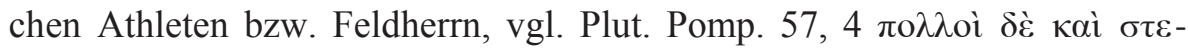

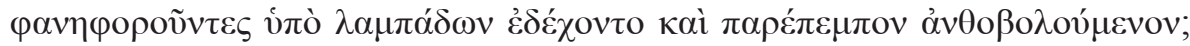

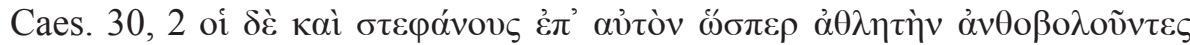

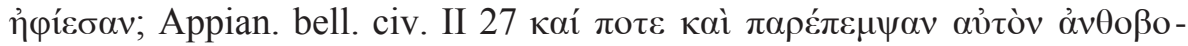

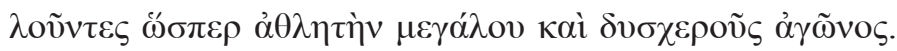

Wenn diese metaphorische Vorstellung (der Kranz als "Blumenwerfer") zu maniriert erscheint, so bietet sich als Konjektur an:

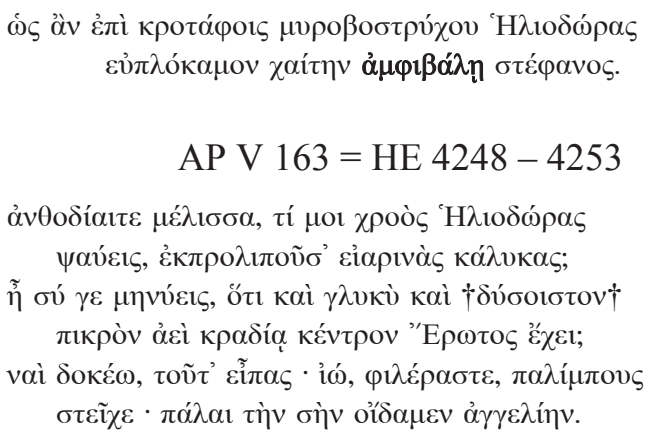

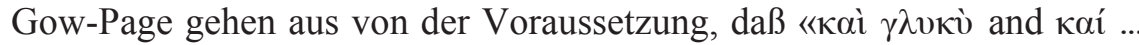

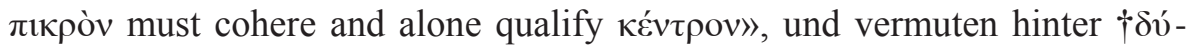

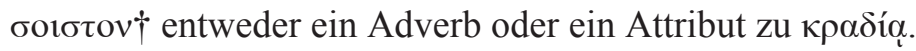


Hiergegen sprechen zwei Bedenken:

1. Inhaltlich. Wie kann man aus der beobachteten Tatsache, daß eine Binne ihre Blüten verläßt und die Haut der Heliodora berührt, schlußfolgern, daß der Stachel der Liebe sowohl süß als auch bitter ist? Wenn die Biene dem Stachel der Liebe entspricht, so wendet sie sich, indem sie die Blüten verläßt, dem "süßen" Aspekt ihrer Tätigkeit ab und belästigt stattdessen Heliodora, möglicherweise in der Absicht, sie

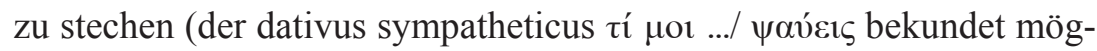

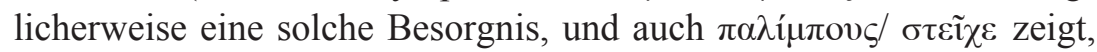
daß die Anwesenheit der Biene nicht gerade erwünscht ist). Dies ist der "bittere" Aspekt, welcher der "süßen" Tätigkeit des Blumensammelns entgegengesetzt ist. Dann sollte das "Süße" dem "Bitteren" im

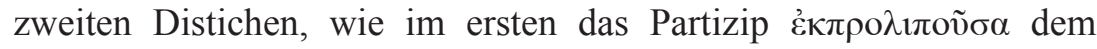

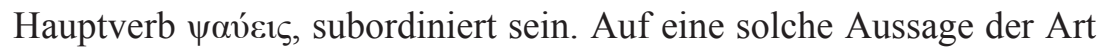

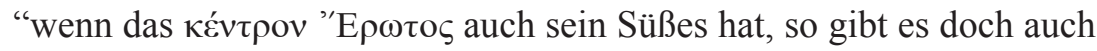
eine bittere Seite", paßt auch das dritte Distichon vorzüglich, in welchem der Sprecher auf seine kummervollen Erfahrungen (wohl mit Heliodora) anspielt.

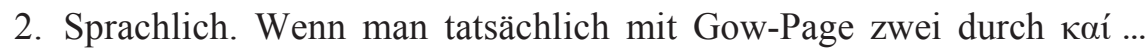

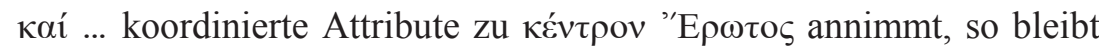
die Konstruktion von "̌́ $\chi \varepsilon ı$ (was ja nicht 'ist' bedeuten kann) völlig unklar.

Die Schwierigkeiten werden mit folgender Textgetsaltung beseitigt:

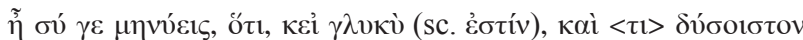

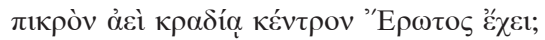

"Bekundest du, daß der Stachel der Liebe, wenn er auch süß ist, doch auch eine schwer erträgliche Bitterkeit stets dem Herzen darbietet $\left(\varepsilon^{\prime} \chi \varepsilon 1=\right.$

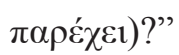

AP V $166=$ HE $4260-4267$

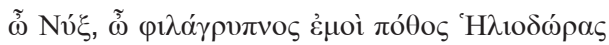

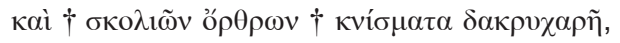

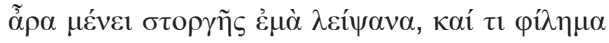

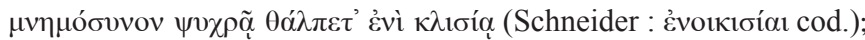

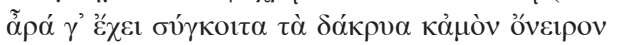




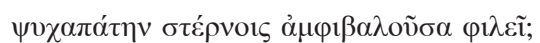

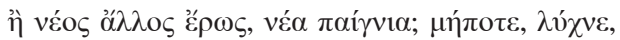

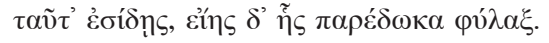

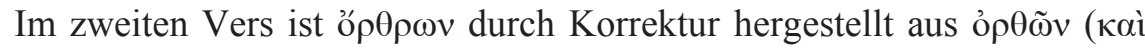

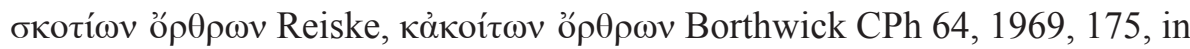
beiden Fällen ein als Genitivattribut kaum zu кví́ $\mu \alpha \tau \alpha$ passender Zeitbegriff). Mit Hilfe von AP V $157=$ HE 4246 f.

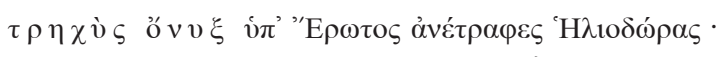

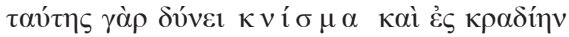

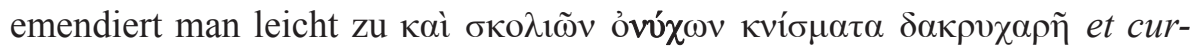
vorum unguium laesiones lacrimas gaudio miscentes (zum Motiv vgl. auch die Stellensammlung bei Nisbet/ Hubbard zu Hor. carm. I 6, 18); solche nächtlichen Raufereien fügen sich bestens nach $\varphi \imath \lambda \alpha ́ \gamma \rho v \pi v o \varsigma$... $\pi$ ó $\theta$ os. Eine ähnliche Deutung schwebte bereits dem bei Jacobs zitierten Manso vor, der diese jedoch mühsam mit dem überlieferten ó $\rho \theta \rho \omega v$ zu vereinbaren suchte: «de uellicationibus et mollibus morsiunculis, quales diluculo infesto ingru-

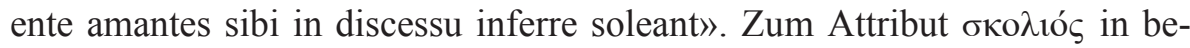
zug auf eine natürliche menschliche Wuchsform von Fingernägeln vgl. Pseu-

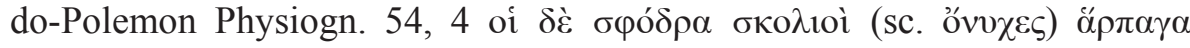

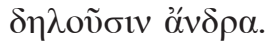

In Vers 3 ist $\dot{\varepsilon} \mu \alpha ́$ Korrektur aus $\dot{\varepsilon} \mu o i ́$. Gow-Page akzeptieren akzeptieren die in griechischer Dichtersprache fragwürdige Enallage für $\sigma \tau о \rho \gamma \eta \tilde{\varsigma} \varsigma \dot{\varepsilon} \mu \tilde{\eta} \varsigma$ $\lambda \varepsilon i ́ \psi \alpha v \alpha$, aber in dem noch vagen Zusammenhang des zweiten Distichons kann man auf das Personalpronomen verzichten und schreiben $\tilde{\alpha} \rho \alpha \mu \varepsilon ́ v \varepsilon 1$

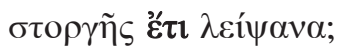

Auch der Fortgang des zweiten Distichons ist über den von Schneider hergestellten Schluß von Vers 3 hinaus korrumpiert. Die Vorstellung eines Kus-

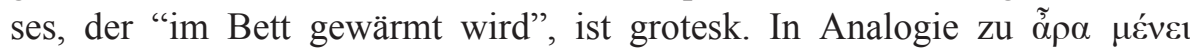

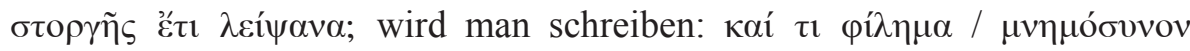

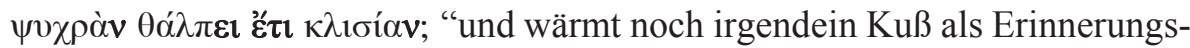
zeichen das kalte Bett?". Zur Hiatkürzung $\theta \alpha \dot{\lambda} \pi \varepsilon \varepsilon$ ह̌ $\tau$ im Pentameter vgl. AP

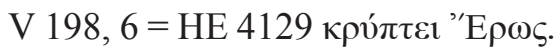

In dem gegenüber dem zweiten Distichon wesentlichen konkreteren $\mathrm{Zu}$ -

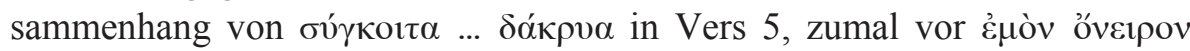
("mein Traumbild", richtig verteidigt von Gow-Page z.St.), wird man dage-

EMERITA (EM) LXXV 1, enero-junio 2007 pp. 93-112 ISSN 0013-6662 


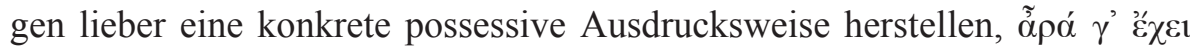

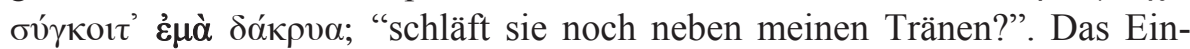
dringen von $\dot{\varepsilon} \mu \alpha ́$ in Vers 3 beruht möglicherweise aus einem Influenzfehler aus Vers 5 .

Im ganzen:

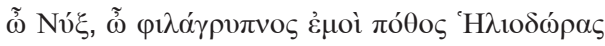

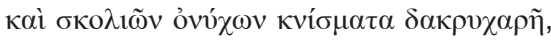

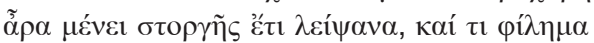

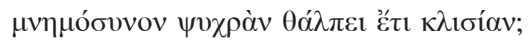

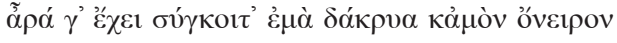

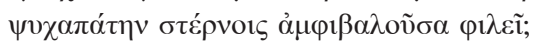

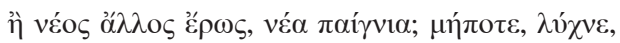

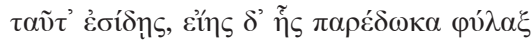

$$
\text { AP V 204, } 1 \text { s. }=\text { HE } 4298 \text { s. }
$$

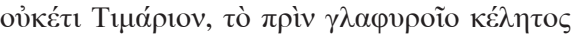

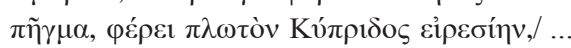

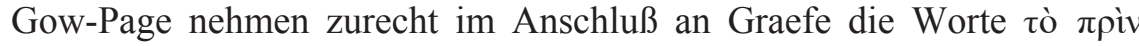

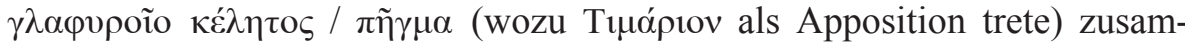
men. Dann ergeben sich aber noch immer Schwierigkeiten mit der Konstruktion von $\pi \lambda \omega \tau$ óv, die zu Konjekturen führten, etwa Meinekes $\pi \lambda \omega \tau \tilde{\omega} v$ oder

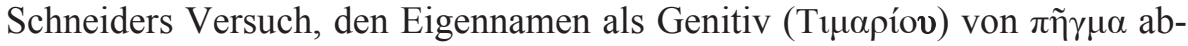

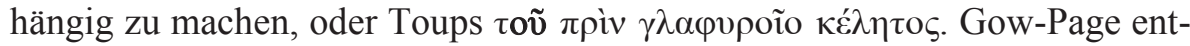
scheiden sich schließlich, $\pi \lambda \omega \tau o ́ v$ als Praedicativum eng mit $\varphi \varepsilon ́ p \varepsilon ı$ zu verbin-

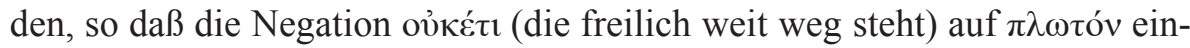
wirken könne.

Es scheint jedoch evident, daß der Begriff $\pi \lambda \omega \tau$ óv in dem den früheren

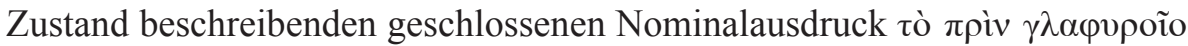
$\kappa \varepsilon ́ \lambda \eta \tau o \zeta / \pi \tilde{\eta} \gamma \mu \alpha$ dringend benötigt wird, um eine Antithese zu dem auf die

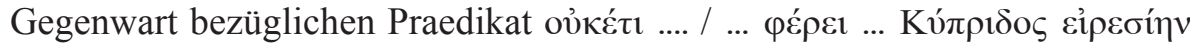
bilden zu können. Dies erreicht man mit einer einfachen Umstellung:

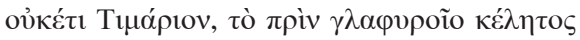

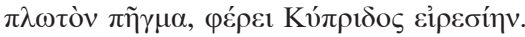




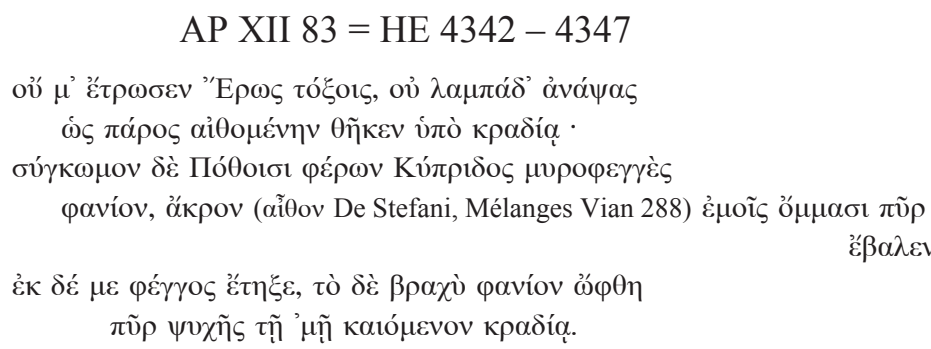

Gow-Page referieren über die vergeblichen Versuche, die Antithese «parua illa fax magnum in pectore meo incendium fecit» (Jacobs) durch konjekturale Abänderung von $\psi v \chi \tilde{\eta} \varsigma$ in den Text einzuführen. Aber der Ausdruck $\pi \tilde{\nu} \rho \psi v \chi \tilde{\eta} \varsigma$ wirkt seinerseits nicht wie eine Korruptel.

Vielleicht sollte man den Begriff der Größe lieber an anderer Stelle ein-

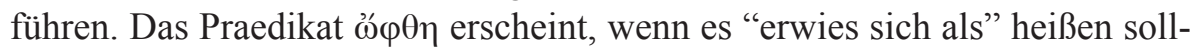
te, ziemlich schwach, zumal das Sehen kurz zuvor noch ein Mittel der eroti-

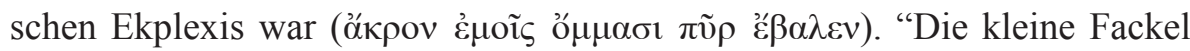

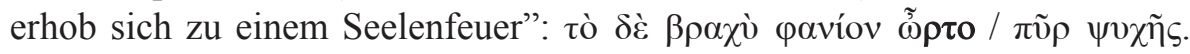

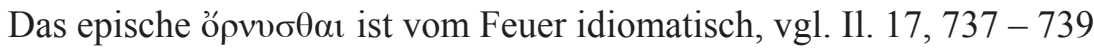

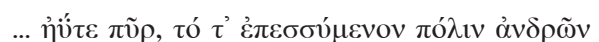

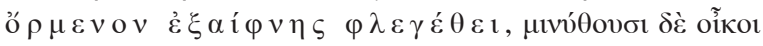

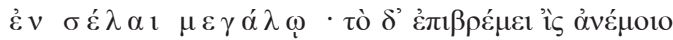

(ferner Soph. Oed. 177). Andererseits paßt öpvvo $\theta \alpha$ auch auf psychische Vorgänge (vgl. LSJ s.v. 3 b), und die Verbindung mit einem Praedicativum ist zumindest in hellenistischer Zeit unproblematisch, wie die abgeblaßte

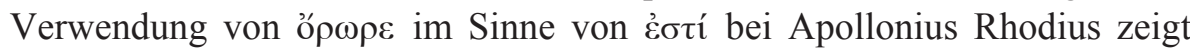
(LSJ s.v. 4).

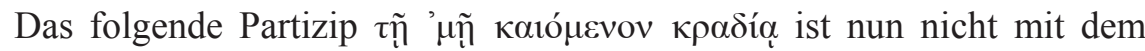
praedikativen $\pi \tilde{v} \rho$ $\psi v \chi \tilde{\eta} \varsigma$ zu verbinden (in diesem Fall müßte sich die be-

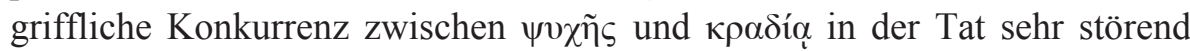

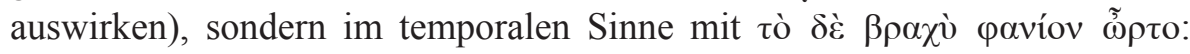
"Die kleine Fackel erhob sich zu einem Seelenfeuer, als sie in meinem Herzen entbrannt wurde". Dadurch, daß die "kleine Fackel" ihre Wirkung erst entfaltet, als sie im Herzen des Sprechers brennt, unterscheiden sich das Vorgehen des Eros in diesem Fall von dem sonst üblichen, wobei eine Fackel in bereits brennendem Zustand in das Herz des Liebenden gelegt wird (ov̉

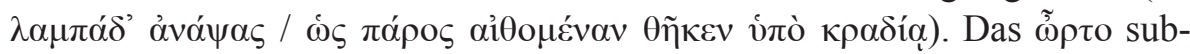

$$
\text { EMERITA (EM) LXXV 1, enero-junio } 2007 \quad \text { pp. 93-112 ISSN 0013-6662 }
$$




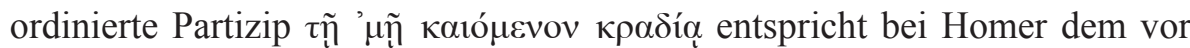

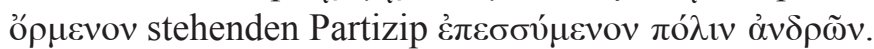

Das gleiche Resultat könnte man mit dem noch wesentlich geringeren Eingriff $̋ ̋ \theta \eta$ erreichen, aber dieser passive Aorist könnte sich ausschließlich auf Korinna PMG 654 col. I 22 ( $\tau$ v̀ $\delta ’ ~ \alpha ́ \mu \alpha ~ \pi \alpha ́ v \tau \varepsilon[\varsigma] ~ \tilde{\omega} \rho \theta \varepsilon v$ ) stützen.

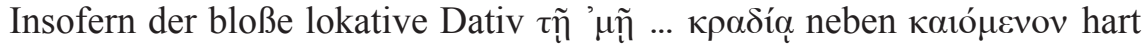
ist und auch eine Auffassung als dativus sympatheticus oder incommodi nicht überzeugt, empfielt es sich, ohne Aphaerese bzw. Krasis einfach zu

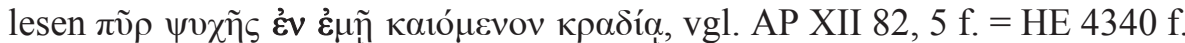

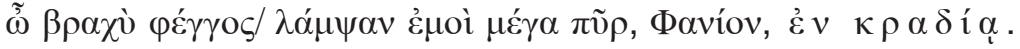

"Die 'kleine Fackel' erhob sich zu einem Seelenfeuer, als sie erst in meinem Herzen angezündet wurde".

Con arreglo a las normas editoriales vigentes para las publicaciones periódicas del CSIC, se hace constar que el original definitivo de este artículo se recibió en la redacción de EMERITA en el primer semestre de 2007, tras haber sido aprobada su publicación en ese mismo período (26.3.07 - 14.01.07) 